ARTICLE

Received 2 Nov 2016 | Accepted 26 Jan 2017 | Published 13 Mar $2017 \quad$ DOl: 10.1038/ncomms14814 OPEN

\title{
Double-stranded RNA virus outer shell assembly by bona fide domain-swapping
}

Zhaoyang Sun ${ }^{1, \star}$, Kamel El Omari ${ }^{1}{ }^{\star}$, Xiaoyu Sun ${ }^{2}$, Serban L. Ilca ${ }^{1}$, Abhay Kotecha ${ }^{1}$, David I. Stuart ${ }^{1}$, Minna M. Poranen ${ }^{2} \&$ Juha T. Huiskonen ${ }^{1,2}$

Correct outer protein shell assembly is a prerequisite for virion infectivity in many multi-shelled dsRNA viruses. In the prototypic dsRNA bacteriophage $\phi 6$, the assembly reaction is promoted by calcium ions but its biomechanics remain poorly understood. Here, we describe the near-atomic resolution structure of the $\phi 6$ double-shelled particle. The outer $T=13$ shell protein P8 consists of two alpha-helical domains joined by a linker, which allows the trimer to adopt either a closed or an open conformation. The trimers in an open conformation swap domains with each other. Our observations allow us to propose a mechanistic model for calcium concentration regulated outer shell assembly. Furthermore, the structure provides a prime exemplar of bona fide domain-swapping. This leads us to extend the theory of domain-swapping from the level of monomeric subunits and multimers to closed spherical shells, and to hypothesize a mechanism by which closed protein shells may arise in evolution.

\footnotetext{
${ }^{1}$ Division of Structural Biology, Wellcome Trust Centre for Human Genetics, University of Oxford, Roosevelt Drive, Oxford OX3 7BN, UK. ${ }^{2}$ Department of Biosciences, University of Helsinki, Viikinkaari 9, Helsinki 00014, Finland. * These authors contributed equally to this work. Correspondence and requests for materials should be addressed to M.M.P. (email: minna.poranen@helsinki.fi) or to J.T.H. (email: juha@strubi.ox.ac.uk).
} 
T he protein shells of icosahedral viruses assemble with remarkable accuracy from smaller subunits ${ }^{1-3}$. Accumulating evidence suggests that such viruses can be grouped into a fairly small number, perhaps less than a dozen, of viral lineages, each sharing a set of common assembly principles 4,5 . However, explaining how such closed, highly symmetric shells may have arisen during evolution poses a challenge, as formation of a closed shell first requires a network of complementary subunit-subunit interactions with roughly $50 \%$ of the surfaces buried from solvent ${ }^{6}$. One of the most complex examples of such interactions is the capsid of bacteriophage $\mathrm{HK} 97$ where the capsid proteins are intertwined to form a topologically linked 'chain mail' organization? A simpler question to ask is how do multimeric proteins evolve from monomers? It has been suggested that this can be achieved by a mechanism called 'domain swapping', ${ }^{8,9}$. In the resulting multimer, the domain-swapped subunits in an 'open' conformation recapitulate the domain-domain interactions of the original 'closed' conformation of the monomer. This concept explains how mutations increasing the flexibility of a linker region of the monomer can facilitate domain swapping to give rise to the multimer.

Domain-swapping may play a role not just in the assembly and evolution of multimeric proteins, but also of viral shells ${ }^{10-12}$. The structure of rice yellow mottle virus (RYMV; genus Sobemovirus), and its comparison to the related southern cowpea mosaic virus (SCPMV), provides an interesting exemplar ${ }^{12}$. In SCPMV, the $T=3$ icosahedral capsid is formed in an usual way by trimers in a compact, closed conformation ${ }^{13}$. In RYMV, however, the trimers are atypical and adopt an open conformation, swapping domains with the neighbouring trimers and creating long range interactions, to which the increased stability of the capsid has been attributed ${ }^{12}$. However, it remains unclear whether RYMV trimers can exist in both open and closed conformations.

We use bacteriophage $\phi 6$, a member of the Cystoviridae family, as a model system to study the assembly of a complicated multishelled virus. The inner $T=1$ shell, composed of protein P1, forms a dodecahedral polymerase complex (PC) harbouring the three viral dsRNA segments $(\mathrm{S}, \mathrm{M} \text {, and } \mathrm{L})^{14,15}$, the viral polymerase P2 (refs 16,17), the hexameric packaging protein P4 (refs 18,19), and the assembly cofactor P7 (refs 20,21). Unlike eukaryotic dsRNA viruses, $\phi 6$ and other cystoviruses undergo a dramatic conformational change during RNA packaging and replication, from an empty collapsed PC to a fully expanded, packaged $\mathrm{PC}^{22,23}$. The expanded $\mathrm{PC}$ is covered by a layer of $\mathrm{P} 8$, forming the outer $T=13$ shell $^{23}$. This double-shelled particle, or nucleocapsid (NC), is itself covered by a protein-lipid envelope during virion assembly ${ }^{24,25}$. A remarkable aspect of the $\phi 6$ system is that PC and NC particles can be assembled in vitro from purified protein and RNA components ${ }^{26}$. In addition to $\phi 6$ bacteriophage (Cystovirus), many other dsRNA viruses, such as aquareovirus (Aquareovirus) ${ }^{27}$, mammalian reovirus (Orthoreovirus) $)^{28}$, blue tongue virus (Orbivirus) $^{29,30}$, rice dwarf virus (Phytoreovirus) ${ }^{31,32}$, rotavirus (Rotavirus) $^{33}$, and Banna virus (Seadornavirus) ${ }^{34}$, form a multishelled particle with an inner $T=1$ and one or more outer $T=13$ shells. While structures for some of these have been solved to near-atomic resolution shedding light into molecular interactions in the $T=1$ and $T=13$ shells ${ }^{27,29,30,33}$, assembly of the $T=13$ shell in $\phi 6 \mathrm{NC}$ has remained elusive.

Here we present the structure of $\phi 6 \mathrm{NC}$ to near-atomic resolution using electron cryomicroscopy and single particle analysis. The structure of the outer NC shell reveals an intricate domain-swapped architecture created by $\mathrm{P} 8$ trimers. The majority of the P8 subunits (540 of 600) are in an open conformation, swapping domains with their neighbours. The inter-trimer
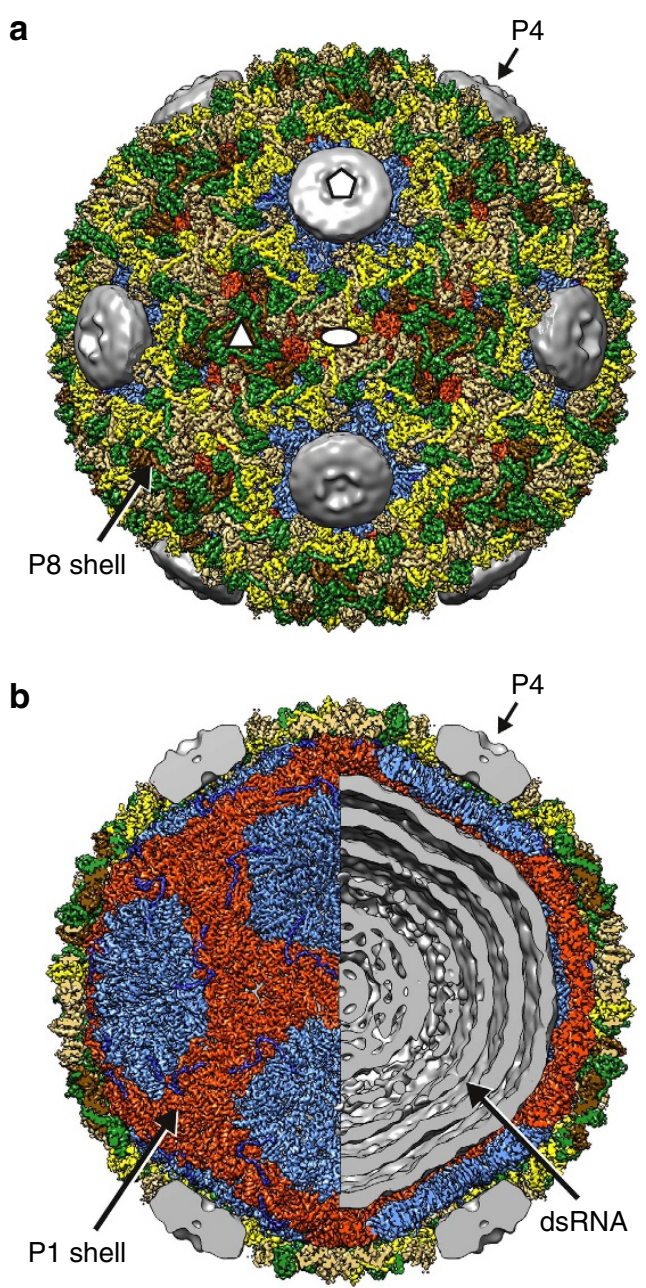

Figure 1 | Cryo-EM structure of bacteriophage $\phi 6$ nucleocapsid.

$(\mathbf{a}, \mathbf{b})$ Three-dimensional reconstruction of the NC at $4.0-\AA$ resolution. The P8 trimers forming the outer protein shell are coloured in yellow $\left(P 8_{Q}\right)$, green $\left(P 8_{R}\right)$, gold $\left(P 8_{S}\right)$ and brown $\left(P 8_{T}\right)$. Half of the $P 8$ density was removed to reveal in $\mathbf{b}$ the inner protein shell composed of $P 1_{A}-P 1_{B}$ asymmetric dimers, coloured in light blue $\left(P 1_{A}\right)$ and red $\left(P 1_{B}\right)$. The elongated density corresponding to the P4 C-terminus is shown in dark blue. Residual density, not assigned to the aforementioned icosahedrally ordered components was filtered to $15-\AA$ resolution to visualize in $\mathbf{a}, \mathbf{b}$ the symmetry-mismatched P4 hexamers at the icosahedral five-fold vertices and the dsRNA in $\mathbf{b}$. See also Supplementary Fig. 1.

interactions recapitulate the intra-trimer interactions of the closed conformation that were also observed within the NC. These observations not only provide a possible explanation for the calcium-regulated assembly of the $\phi 6 \mathrm{NC}$ particle, but they also constitute an exemplar of bona fide domain swapping in a viral shell, allowing us to propose a mechanism for how such complex shells may have arisen during evolution.

\section{Results}

Near-atomic resolution structure of the $\phi 6$ nucleocapsid. We determined the structure of $\phi 6$ double-shelled particle by electron cryomicroscopy (cryo-EM; Fig. 1; Table 1). A highresolution cryo-EM data set was acquired by imaging purified ф6 NC samples, some of which had spontaneously lost the RNA genome (Supplementary Fig. 1a). Two-dimensional image classification revealed the morphology of NC particles 
Table 1 | Nucleocapsid cryo-EM data collection parameters.

\section{Parameter}

Voltage (kV)

Magnification $(x)$

Defocus $(\mu \mathrm{m})$

Dose rate ( $e^{-}$per pixel per $s$ )

Frames

Frame length (s)

Total dose ( $e^{-}$per $\AA^{2}$ )

Micrographs

cryo-EM, electron cryomicroscopy.

(Supplementary Fig. 1b). Some particles had lost either fully or partially the outer P8 shell (Supplementary Fig. 1a,b). Complete double-shelled particles were used to calculate a three-dimensional reconstruction using a 'gold-standard' refinement approach (see Methods; Table 1). The overall resolution of the icosahedrally symmetric areas of the reconstruction was $4.0 \AA$, as determined by Fourier shell correlation (FSC, threshold $=0.143$; Supplementary Fig. 1c). In many areas the local resolution was approaching $3.0 \AA$, while some areas, especially solvent exposed loops and areas around the icosahedral five-fold vertices in the outer protein shell were resolved to a lower resolution (Supplementary Fig.1d-i). The resolution was sufficient to not only trace the course of the polypeptide chain but also to align the sequence to the structure and refine the structure, for nearly all of P1 and P8 residues (see Methods). The P4 hexamers located at the icosahedral five-fold axes of symmetry were not resolved due to the symmetry mismatch (Fig. 1). Density corresponding to the three linear dsRNA genome segments appeared as concentric layers spaced $\sim 3 \mathrm{~nm}$ apart but the detailed organization of the segments could not be addressed using icosahedral reconstruction (Fig. 1b).

Architecture of the nucleocapsid outer shell. The NC structure revealed, consistent with our earlier observations ${ }^{23}$, a total of 200 P8 trimers forming the outer protein shell, following $T=13$ laevo quasi-symmetry (Fig. 1a). Four types of trimers $\left(\mathrm{P} 8_{\mathrm{Q}}, \mathrm{P} 8_{\mathrm{R}}, \mathrm{P} 8_{\mathrm{S}}\right.$ and $\left.\mathrm{P} 8_{\mathrm{T}}\right)$ occupy the different positions in the shell: Q-type trimers around the icosahedral five-fold axis of symmetry, S-type trimers adjacent to the two-fold axis, T-type trimers on the three-fold axis and R-type trimers between the other trimers. Each of the 60 asymmetric units of the shell thus consists of $10 \mathrm{P} 8$ chains, as the $\mathrm{P} 8_{\mathrm{T}}$ trimers contribute only one chain to each asymmetric unit.

The $T=13$ shell revealed a remarkable domain swapped organization of P8 trimers (Fig. 2). The relatively high resolution of the reconstruction allowed modelling the fold of the P8 structure (residues 3-147; Fig. 2a,b; Supplementary Fig. 2). The structure is $\alpha$-helical and can be divided into two domains that are connected by an elongated linker (Fig. 2c,d): an $\mathrm{N}$-terminal peripheral domain ( $\mathrm{PD}$; residues 1-83) is followed by a 10-residue long elongated loop (residue 84-93) and a C-terminal core domain (CD; residues 94-149). The loop ends in a proline residue (Pro93), which breaks the preceding helix $\alpha 6$ of the CD (Supplementary Fig. 2). The CDs intertwine together to form the core of the P8 trimer. The PDs, due to the elongated nature of the linker, are located a considerable distance ( $\sim 50 \AA$ ) from the CD. Instead of interacting with the CD of the trimer they belong to, for the majority of the P8 molecules the PDs swap between the trimers, each PD interacting with the CDs and PDs of adjacent trimers via heterotypic interactions
(Fig. 2e,f). Nine such structures are observed in the icosahedral asymmetric unit of the NC.

In addition to the 'open' conformation described above and adopted by 9 out of 10 of the P8 chains, a fundamentally different conformation is also found on the shell. This 'closed', more compact, conformation is observed in one of the three chains of the peripentonal $\mathrm{P} 8_{\mathrm{Q}}$ trimer (Fig. 2g; Supplementary Fig. 2). The two conformations differ in degree of bending of the elongated linker that acts as a hinge. The change in relative position between the two conformations is $\sim 75 \AA$ and involves a rotation of 71 degrees (Fig. 2h). In the closed conformation, the $\mathrm{PD}$ is bound to its own $\mathrm{CD}$ via a homotypic interaction (Fig. 2i). This interaction is characterised by an interface that is analogous to the one created between the $\mathrm{PD}$ and a $\mathrm{CD}$ of an adjacent trimer in heterotypic interactions (Fig. 2j).

P4 hexamer is anchored to the vertices of the P1 shell. In the members of the subfamily Sedoreovirinae of the family Reoviridae, such as blue tongue virus (Orbivirus) ${ }^{29}$ and rice dwarf virus (Phytoreovirus) ${ }^{32}$, P-type trimers occupy the five-fold vertices. In contrast, in members of Cystoviridae, such as $\phi 6$ (ref. 23) and Pinareovirinae, such as aquareoviruses ${ }^{27}$, the P-type trimers are absent leaving room for turret-like structures at the icosahedral five-fold vertices. In $\phi 6$, the vertices are occupied by the $\mathrm{P} 4$ packaging hexamer (Fig. 1). The presence of $\mathrm{P} 4$ hexamers at the five-folds provides also a rationale for the observed closed conformation of certain peripentonal $\mathrm{P} 8$ proteins (Supplementary Fig. 3); if the type $\mathrm{P} 8_{\mathrm{Q}}$ monomers were all in the open conformation, significant clashes would occur between $\mathrm{P} 8_{\mathrm{Q}}$ and $\mathrm{P} 4$ (Supplementary Fig. 3b).

The six-fold symmetry of the $\mathrm{P} 4$ hexamer and five-fold symmetry of the icosahedral vertex beneath creates a symmetry mismatch, so that the structure of $\mathrm{P} 4$ cannot be resolved using conventional icosahedral reconstruction ${ }^{23}$. To address this we applied the localized reconstruction method ${ }^{17}$ to P4 hexamers. Local areas corresponding to P4 hexamers were extracted from the NC images and reconstructed as single particles with six-fold symmetry ('P4 hexamer reconstruction') or without any symmetry ('NC vertex reconstruction'). This allowed a low-resolution structure of the $\mathrm{P} 4$ hexamer to be determined in situ (Fig. 3). At a resolution of 9.1 $\AA$ ( $F S C=0.143)$, fitting of a P4 crystal structure (PDB:4BLO) ${ }^{19}$ into the density was unambiguous (Fig. $3 \mathrm{a}-\mathrm{c}$ ) and allowed us to orient the hexamer relative to the NC vertex (Fig. 3d,e).

A disorder prediction algorithm suggests that the C-terminal residues of $\mathrm{P} 4$ are likely to be disordered (Supplementary Fig. 4$)^{35}$. Indeed, they are missing in the crystal structure and this disordered region has been proposed to attach the $\mathrm{P} 4$ to the underlying P1 shell ${ }^{19}$. However, in earlier low-resolution cryo-EM reconstructions, the P4 hexamers appeared to float on top of the five-fold vertexes of the P1 shell ${ }^{23,36,37}$ and the connections between these symmetrymismatched components have remained unresolved. Our localized reconstruction of $\mathrm{P} 4$ hexamers that correctly dealt with the symmetry mismatch revealed elongated densities extending from 3 of the 6 P4 C-terminal parts (Fig. 3f). We hypothesised that these may correspond to the C-terminal parts that are disordered in the crystal structure. Consistent with this hypothesis, in the high-resolution icosahedral NC reconstruction we observed sixty copies of elongated density that lacked any apparent secondary structure stretching $\sim 115 \AA$ on top of each of the asymmetric P1 dimers (Figs $1 \mathrm{~b}$ and $4 \mathrm{c}-\mathrm{e})$. At the vertex distal part of this density, densities consistent with the side-chains 324-ProArgArg-326 


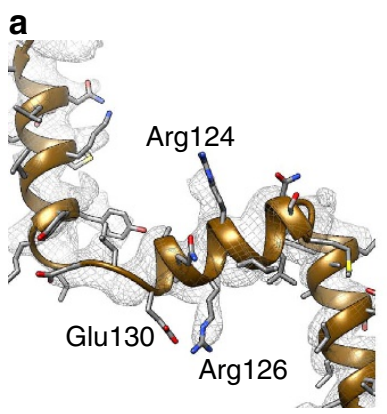

b

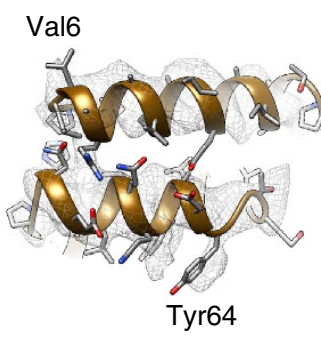

c

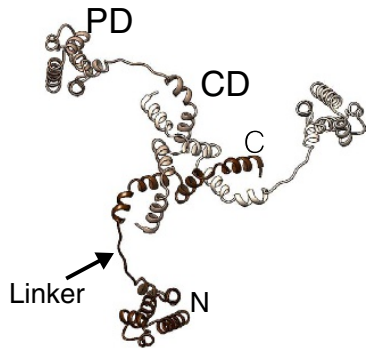

d

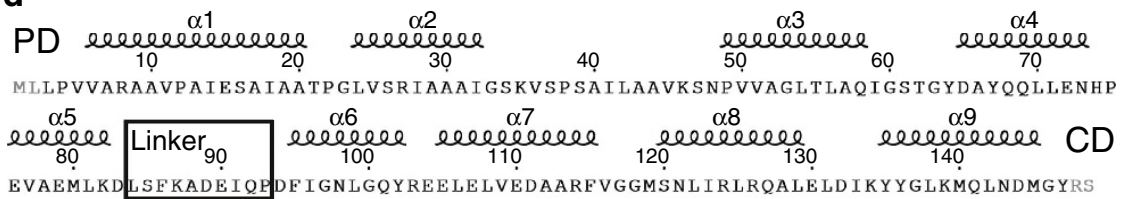
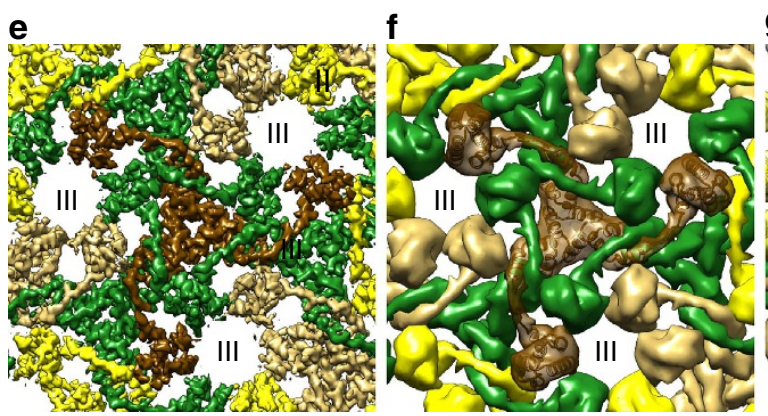

g

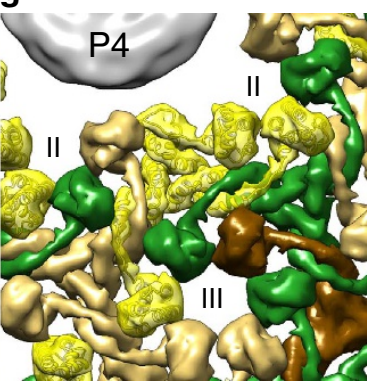

h

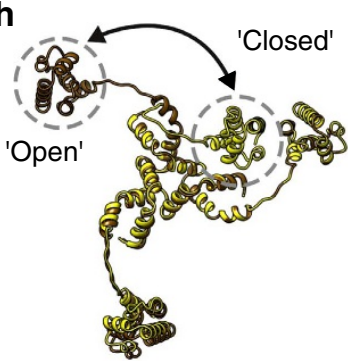

i
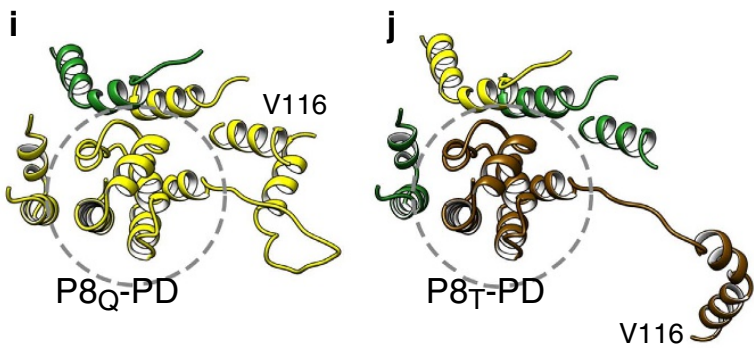

Figure 2 | Domain swapped architecture of the outer $\mathbf{\phi 6} \mathbf{P 8}$ shell. $(\mathbf{a}, \mathbf{b})$ Close-ups of the atomic model of the T-type trimer (P8 $8_{T}$ ) and corresponding density (mesh). Some side chains are labelled. (c) Atomic model of the P8 trimer. The three different chains are shown in different shades. The peripheral domain (PD) and the core domain (CD) are labelled and the corresponding areas of the structure circled. The linker (arrow), $N$ terminus (N) and C terminus (C) are labelled for one chain (dark brown). (d) Sequence of the P8 with secondary structure elements, PD, CD and the linker region labelled. Amino-acid residues not resolved in the structure are in grey. (e) A close-up of the P8 shell density viewed along the three-fold axis of symmetry. The linkers from different trimers intertwine, leading to domain swapping of the peripheral domains between the trimers. (f) Same view as in $\mathbf{e}$ but a rough molecular surface is shown for clarity. $\mathrm{P} 8_{\mathrm{T}}$ is shown as a ribbon. ( $\mathbf{g}$ ) Same as $\mathbf{f}$ but a view showing the Q-type trimers ( $\mathrm{P} 8_{\mathrm{Q}}$, ribbon) around the P4 hexamers. P4 is shown as a grey surface. (h) Structural alignment of $\mathrm{P} 8_{\mathrm{T}}$ and $\mathrm{P} 8_{\mathrm{Q}}$ trimers. The location of the peripheral domain in 'open' and 'closed' conformation of the trimer is indicated. (i) The mode of interaction of $\mathrm{P} 8_{\mathrm{Q}}-\mathrm{PD}$ in closed conformation is shown. The interactions are mainly of homotypic nature with other parts of the same trimer. (j) The mode of interaction of the P8 $8_{T}-P D$ in open conformation is shown. The interactions are of heterotypic nature with other types of trimers. The $\mathrm{P} 8$ trimers are coloured in yellow $\left(\mathrm{P} 8_{\mathrm{Q}}\right)$, green $\left(\mathrm{P} \mathrm{8}_{\mathrm{R}}\right)$, gold $\left(\mathrm{P} 8_{\mathrm{S}}\right)$ and brown $\left(\mathrm{P} 8_{\mathrm{T}}\right)$ and the type II and type III holes 23 are labelled. See also Supplementary Figs 2 and 3.

of the P4 C terminus were identified (Fig. 4f), allowing us to specifically assign this density to the C-terminal residues 292-332 of $\mathrm{P} 4$. The sixth, unbound C-terminal region of $\mathrm{P} 4$ was not resolved in any of the reconstructions, and is likely to be either disordered or unresolved in our maps due to limited resolution of the localized reconstructions.

Binding of up to five of the six flexible C-terminal tails of $\mathrm{P} 4$ provides an elegant solution to the symmetry mismatch. These tails are likely to be disordered in solution (Supplementary Fig. 4) and to order only upon binding to P1 during assembly.
The P4 tails are analogous to the 'stay-cables' observed earlier in adenovirus fibres ${ }^{38}$. As such cables have now been observed in at least two unrelated viruses, adenovirus (a dsDNA virus ${ }^{38}$ and $\phi 6$ (a dsRNA virus, this study), they may be a general solution to the problem of attaching fibres and other symmetry-mismatched appendices to vertices of icosahedral virus capsids.

P4 C-terminal tail solidifies the dimeric P1 building block. The inner P1 shell is composed of 60 copies of asymmetric P1 dimers, 
a

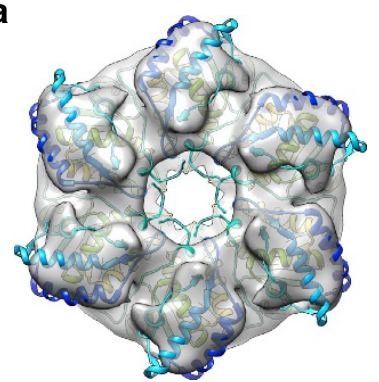

b

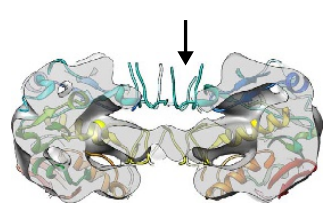

C

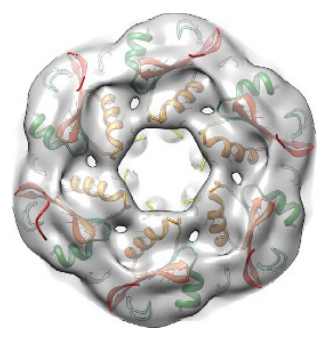

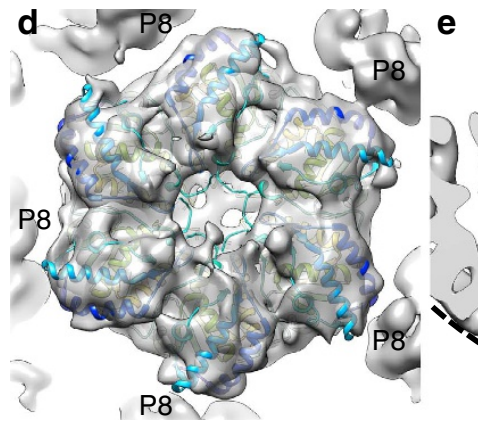

e
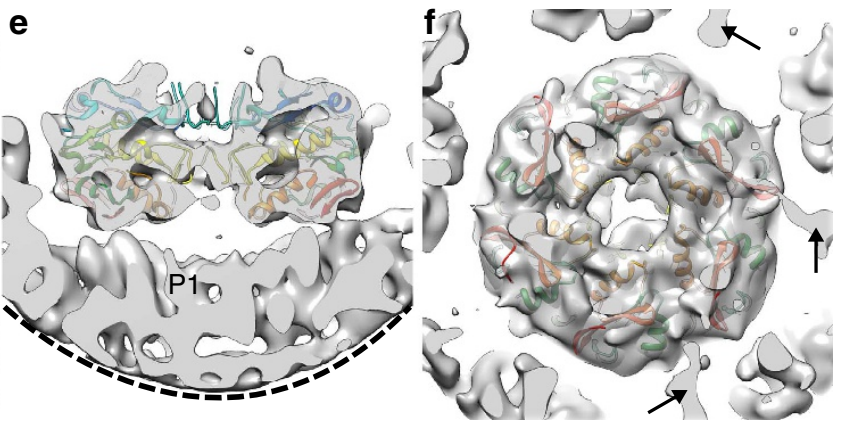

Figure 3 | Hexameric packaging ATPase P4. (a-c) Localized reconstruction of $\phi 6$ P4 with six-fold symmetry is shown as a grey transparent surface from the top (a), side (b) and below (c). P4 crystallographic structure ${ }^{19}$ is coloured from red (C-terminus) to blue ( $\mathrm{N}$-terminus) and was fitted into the reconstruction as a rigid body. Flexible loops that were only partially resolved in the crystallographic structure were not resolved in the localized reconstruction (arrow). (d-f) Same views as in a-c showing the localized reconstruction without symmetry. The edge of a mask cutting through the density under the P4 hexamer is indicated with a dashed line in e. Three densities possibly connecting the P1 shell to the P4 C-termini are indicated with arrows.

formed by $\mathrm{P} 1_{\mathrm{A}}$ and $\mathrm{P} 1_{\mathrm{B}}$ (see Fig. 1b) ${ }^{23}$. A large-scale expansion of the P1 shell occurs during RNA packaging to empty particles and subsequent RNA replication ${ }^{22,23}$. To model the structural transitions in the $\mathrm{P} 1$ shell during this expansion, we refined the existing atomic coordinates of the P1 monomer (PDB:4K7H $)^{39}$ into our EM density map of the NC (Fig. 4a,b) and compared them to P1 coordinates flexibly fitted to an unexpanded $\mathrm{PC}$ reconstruction (PDB:4BTG) ${ }^{39}$. The angle between the two subunits in the $\mathrm{P} 1_{\mathrm{A}}-\mathrm{P} 1_{\mathrm{B}}$ asymmetric dimer changes relatively little $\left(11^{\circ}\right)$ showing that the $\mathrm{P} 1_{\mathrm{A}}-\mathrm{P} 1_{\mathrm{B}}$ dimer behaves as quite a rigid block during the expansion (Supplementary Fig. 5a,b; Supplementary Movie 1). In contrast to these intradimer angles, the interdimer angles change significantly, mainly at the $\mathrm{P} 1_{\mathrm{B}}-\mathrm{P} 1_{\mathrm{B}}$ interfaces ${ }^{39}$. The interdimer angle changes $57^{\circ}$ at the two-fold interface and $49^{\circ}$ at the three-fold interface (Supplementary Fig. $5 c, d$ ). The root-mean-square deviation (RMSD) calculated between the two states was 2.6 and $6.0 \AA$ for the $\mathrm{P} 1_{\mathrm{A}}$ and $\mathrm{P} 1_{\mathrm{B}} \mathrm{C}$-alpha coordinates, respectively. This shows that relatively small changes occur within the $\mathrm{P} 1_{\mathrm{A}}$ subunits, while $\mathrm{P} 1_{\mathrm{B}}$ subunits adjust to their local environment in the two very different $\mathrm{P} 1$ shell expansion states.

The relative rigidity of the $\mathrm{P} 1_{A}-\mathrm{P} 1_{B}$ dimer interface is now explained by our observation that the $\mathrm{C}$-terminal part of $\mathrm{P} 4$ bridges the $\mathrm{P} 1_{\mathrm{A}}$ and $\mathrm{P} 1_{\mathrm{B}}$ monomers (Fig. 4c,d) whilst still allowing interdimer angles to change. Thus, the P4 C-terminus seems not only to link P4 hexamers to the P1 shell, but also to play a role in P1 dimerization. A dimer of the major inner capsid protein could indeed be a conserved intermediate in the assembly pathways of dsRNA viruses. This notion is supported by the structure of the Penicillium chrysogenum virus in which the equivalent building block is formed by a covalently linked major capsid protein dimer, produced by gene duplication ${ }^{40}$. In $\phi 6$, we propose that the $\mathrm{C}$-terminal tail of $\mathrm{P} 4$ provides an analogous linkage.

\section{Discussion}

When combined with earlier structural and biochemical results, our NC structure allows us to propose an assembly model for $\phi 6$ (Fig. 5; Supplementary Movie 2). The assembly of the P1 shell is initiated in vitro by $\mathrm{P} 4$ hexamers ${ }^{26}$ and the C-terminus of $\mathrm{P} 4$ is essential for the formation of recombinant PC in vivo ${ }^{41}$. We now have a structural rationale for these observations, since the P4 C-terminus cements the P1 asymmetric dimer together (Fig. 4c,d). Accordingly, we suggest that the $\$ 6$ PC assembly starts with the formation of a $\mathrm{P}_{\mathrm{A}}-\mathrm{P} 1_{\mathrm{B}}$ dimer stabilized by the C-terminus of P4 (Fig. 5a). As observed in the P1 crystal structure, individual P1 chains tend to create a pentamer in a conformation that resembles a group of five $\mathrm{P} 1_{\mathrm{A}}$ chains in the unexpanded conformation of $\mathrm{PC}^{39}$. Our modelling experiments also showed that six $\mathrm{P}_{\mathrm{A}}-\mathrm{P} 1_{\mathrm{B}}$ dimers per $\mathrm{P} 4$ would create steric clashes (Supplementary Movie 2). It is thus likely that each $\mathrm{P} 4$ grabs five and not more $\mathrm{P} 1_{\mathrm{A}}-\mathrm{P} 1_{\mathrm{B}}$ dimers. Dimers attached to distinct $\mathrm{P} 4$ hexamers then interact to eventually form a closed P1-shell (Fig. 5b). The assembly of the compact empty PC is facilitated by the assembly cofactor P7 (refs 20,26). P7, together with $\mathrm{P} 2$, is located at the three-fold symmetry axis of the empty $\mathrm{PC}^{21}$, but the order of $\mathrm{P} 2$ and $\mathrm{P} 7$ binding to the $P C$ remains unclear. The $P C$ subsequently packages and replicates the RNA genome segments leading to expansion of the PC (Fig. 5c) 23,39 and finally formation of the double-shelled particles by incorporation of the P8 layer (Fig. $5 \mathrm{~d}$ ).

P8 shells form spontaneously in the presence of $0.1-1.0 \mathrm{mM}$ $\mathrm{Ca}^{2+}$ and in the absence of any other structural proteins ${ }^{42}$. Conversely, depleting calcium by a chelating agent or exposure to low $\mathrm{pH}$ leads to $\mathrm{P} 8$ shell disassembly ${ }^{42,43}$. Raman spectroscopy has revealed that the assembled shell stabilizes the mostly $\alpha$-helical conformation of P8 (ref. 44). It is thus clear that $\mathrm{Ca}^{2+}$ regulates the conformational state of $\mathrm{P} 8$. In the light of our observation of a closed and open state of the P8 trimer in 
a
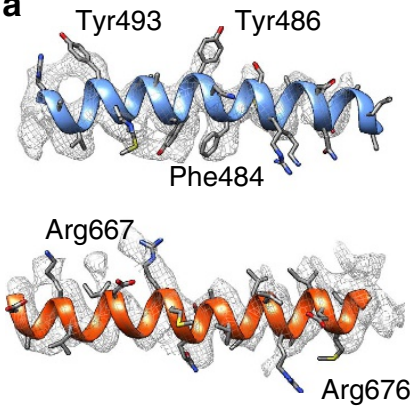

C

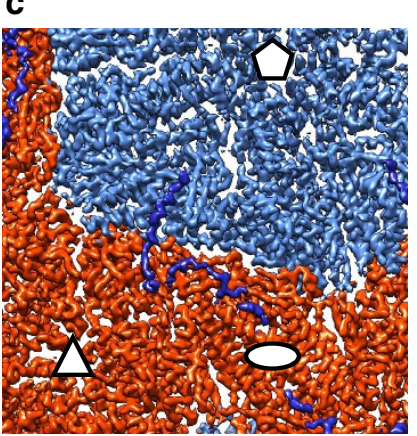

e

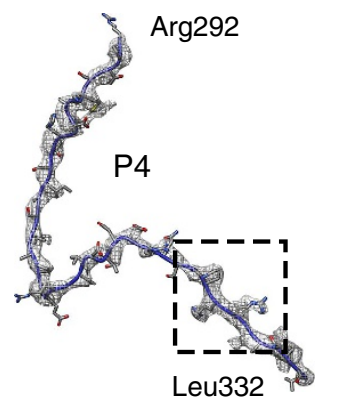

b

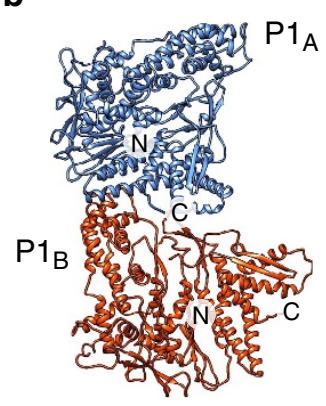

scattering (Z.S. and J.T.H., unpublished observations). The P8 CD domain is preceded by Pro93 that breaks the helix $\alpha 6$ of the CD. Proline residues are abundant in the linker regions of domain swapped proteins and may play a role in modulating the equilibrium between an open and a closed state ${ }^{45}$. It is thus possible that Pro93 has a functional role in adding strain to the backbone of the linker region but this hypothesis remains to be tested.

The observed P8 shell assembly allows us to extend the concept of domain swapping to viral shells ${ }^{8-12}$. Domain swapping is considered bona fide, when the same subunit can still adopt both closed and open conformations, as opposed to the subunits adopting just the open conformation in a multimer and the closed conformation in a different, albeit orthologous, monomeric protein $^{8}$. Following this nomenclature, our $\phi 6$ outer shell structure provides an exemplar of bona fide domain swapping between P8 trimers that exhibit both closed and open conformations.

Analogously to monomer to multimer transition during the evolution of multimeric proteins, our results allow us to propose a hypothetical model how closed viral protein shells may further evolve from such proteins. A primordial, relatively simple capsomer may have some tendency to form larger assemblies, but initially the protein-protein interfaces required to make a spherical shell are not optimized. Mutations in a linker region can increase its flexibility to allow for the formation of an open conformation of the multimeric capsomer. These capsomers in their open conformations would then be able to further link together by recapitulating the domain-domain interactions of the closed conformation. A pre-existing inner protein shell, genome, or internal lipid bilayer present in some viruses, may play a role in increasing the fidelity of shell assembly ${ }^{46}$. This is also evident in the case of $\phi 6$, where in the absence of the inner P1 shell, P8 self-assembles in vitro into aberrant, shell-like structures that vary in size and are not topologically closed ${ }^{43,47}$. In some viruses, the capsomers may have subsequently lost the ability to form both open and closed conformations. To what extent the outer protein shells of $\phi 6$ and other dsRNA viruses are homologous remains an open question, as domain-swapping is not observed in other dsRNA viruses. It is conceivable, however, that linker region deletions and domain-shuffling may have played a further role in stabilizing protein shells. In the case of $\phi 6$, the ability of P8 to still adopt both conformations may have been preserved in evolution as this may play a role in calcium-regulated outer shell assembly/disassembly and also the presence of $\mathrm{P} 4$ hexamer at the vertices necessitates a closed conformation of P8.

In conclusion, our structural characterization of the $\phi 6$ doubleshelled particle revealed interactions of the inner P1 shell and symmetry-mismatched P4 packaging hexamers. Most importantly, the study led to the discovery of two states of the outer shell forming protein P8, 'open' and 'closed'. Trimers in the 'open' conformation swap domains between each other. This process is possibly regulated by calcium ion concentration but further studies are required to reveal the exact mechanism of $\mathrm{Ca}^{2+}$ regulation. The derived outer shell assembly model not only provides insights into assembly in this particular model system, but also suggests how closed protein shells may have arisen during evolution. Further studies are required to assess to what extent domain swapping may have played a role in other viral systems. (Supplementary Fig. 3). $\mathrm{Ca}^{2+}$ regulation of $\mathrm{P} 8$ conformatic possibly in addition to acidic $\mathrm{pH}$, might also be important to facilitate the delivery of the PC to the host cell upon infection.

The conformational space sampled by P8 trimers in solution and at different $\mathrm{Ca}^{2+}$ concentrations remains unknown. Insolubility of purified P8 and its tendency to readily form large assemblies even in the presence of $\mathrm{Ca}^{2+}$-chelating agents has impeded its solution structure studies by small angle X-ray

\section{Methods}

Cryo-EM sample preparation. Bacteriophage $\phi 6$ was grown on its host Pseudomonas syringae pv.phaseolicola $\mathrm{HB} 10 \mathrm{Y}^{48}$ and purified as described previously ${ }^{49}$. The viral envelope was removed by Triton X-114 extraction ${ }^{49}$ and the resulting aqueous mixture containing the NC loaded onto a CIM DEAE-1 


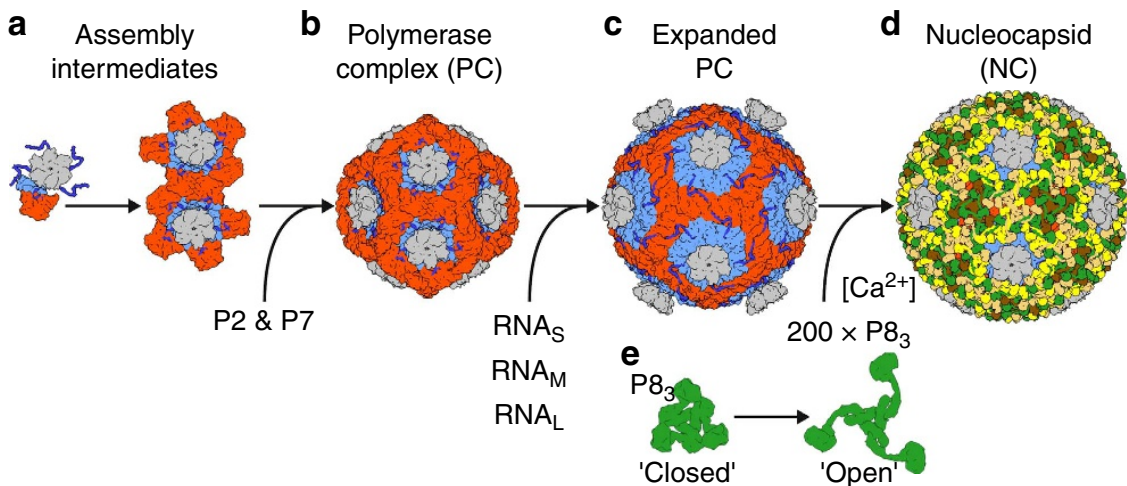

Figure 5 | Assembly model. (a) The $\phi 6$ inner capsid is composed of protein P1, P2, P4 and P7. Preassembled P4 hexamers (grey) nucleate the assembly of the $\mathrm{P} 1$ shell by bringing together two $\mathrm{P} 1$ subunits that will form the asymmetric $\mathrm{P} 1_{A}-\mathrm{P} 1_{B}$ dimer (light blue and red) in the final assembly. The dimerization of $\mathrm{P} 1$ is facilitated by the C-terminal tail of P4 (dark blue). Up to five P1 dimers can assemble around a P4 hexamer. (b) P1 dimers connected to distinct P4 hexamers interact bringing together the precursors of the icosahedral vertices. This is facilitated by protein P7, which acts as an assembly cofactor. (c) The self-assembled empty capsids package and replicate the single-stranded RNA precursors of the $\phi 6$ dsRNA genome (S, M and L) and the capsid adopts the expanded conformation. (d) Trimers of P8 (green, yellow, gold and brown) then assemble around the dsRNA containing inner capsid to form the nucleocapsid. (e) The assembly of the P8 shell is induced by $\mathrm{Ca}^{2}+$ ions and involves major conformational changes in the linker regions of P8 subunits to mediate the transition from the closed to the open conformation. See also Supplementary Movie 2.

Table 2 | Data processing parameters.

\begin{tabular}{lccc} 
Parameter & NC particle & P4 hexamer & NC vertex \\
\hline Particles $^{\star}$ & $13,291(16,466)$ & $56,448(159,492)$ & $56,448(159,492)$ \\
Box size (pixels) & 512 & 128 & 128 \\
Pixel size $(\AA)$ & 1.35 & 1.35 & 1.35 \\
Symmetry & 11 & 66 & $C 1$ \\
Resolution $(\AA)$ & 4.0 & 9.1 & 9.1 \\
B-factor $\left(\AA^{2}\right)^{\dagger}$ & -177 & $-600^{\ddagger}$ & $-600^{\ddagger}$ \\
\hline
\end{tabular}

NC, nucleocapsid.

${ }^{\star}$ Number of particles (or subparticles in the case of P4 hexamer and NC vertex) chosen for averaging the final map is given. The number of all particles extracted from the micrographs (or all subparticles extracted from particles) is given in parenthesis.

†Inverse B-factor used for sharpening of the map.

$\ddagger$ Value of B-factor determined ad hoc

tube monolithic column (BIA Separations, Slovenia) in $20 \mathrm{mM}$ potassium phosphate $\mathrm{pH} 7.2,1 \mathrm{mM} \mathrm{MgCl} 2,0.1 \mathrm{mM} \mathrm{CaCl}_{2}, 150 \mathrm{mM} \mathrm{NaCl}$ from which the bound NC particles were eluted using a $0.15-2 \mathrm{M} \mathrm{NaCl}$ complex gradient (in $20 \mathrm{mM}$ potassium phosphate $\mathrm{pH} 7.2,1 \mathrm{mM} \mathrm{MgCl} 2,0.1 \mathrm{mM} \mathrm{CaCl}$ ). The $\mathrm{NC}$ containing fractions were concentrated using an ultra-centrifugal filter with a $100-\mathrm{kDa}$ cutoff (Amicon; EMD Millipore, Billerica, MA, USA). A 3- $\mu \mathrm{l}$ aliquot of sample, diluted in buffer $\left(20 \mathrm{mM} \mathrm{KPO}_{4}, \mathrm{pH} 7.2,1 \mathrm{mM} \mathrm{MgCl} 2,0.1 \mathrm{mM} \mathrm{CaCl} 2\right.$ $150 \mathrm{mM} \mathrm{NaCl}$ ) to concentration of $3 \mathrm{mg} \mathrm{ml}^{-1}$, was applied to a glow-discharged grid (C-flat; Protochips, Raleigh, NC) and vitrified by plunge-freezing into liquid ethane using a Vitrobot (FEI, Hilsboro, OR).

Data acquisition and processing. Data were acquired using a $300-\mathrm{kV}$ transmission electron microscope (Tecnai F30 'Polara'; FEI) equipped with an energy filter (slit width $20 \mathrm{eV}$; GIF Quantum LS, Gatan, Pleasanton, CA) and a direct electron detector (K2 Summit, Gatan). Movies (22 frames, each frame $0.2 \mathrm{~s}$ ) were collected at in electron counting mode at dose rate of $5 \mathrm{e}^{-}$per pixel per $\mathrm{s}$ at calibrated magnification of $37,037 \times$ resulting in a total dose of $\sim 16 \mathrm{e}^{-}$per $\AA^{2}$ and pixel size of $1.35 \AA$. Data acquisition statistics are summarized in Table 1.

Movie frames were aligned to account for $\mathrm{drift}^{50}$ and contrast transfer function (CTF) parameters were estimated ${ }^{51}$. A total of $\sim 900$ movies, showing minimal drift and astigmatism were used to pick 16,466 particles. Particles were extracted for 2D reference free classification in Relion 1.3 (ref. 52). Particles from classes showing complete P1 and P8 layers were selected for 3D classification using a previously published NC structure (EMD-1,206) as an initial model. A subset of 13,291 particles extracted from movies was refined using standard refinement and particle polishing protocols implemented in Relion.

The structure of the $\mathrm{P} 4$ hexamer was calculated using localized reconstruction (www.opic.ox.ac.uk/localrec) ${ }^{17}$. Briefly, twelve 'subparticles', each corresponding to a projection of the $\mathrm{P} 4$ hexamer in each of the particle images, were extracted (total of 159,463 subparticles) to a smaller box and treated as independent single particles. To assign the orientation for each subparticle, one of the five possible triplets of Euler angles that were equivalent for the corresponding five-fold symmetric vertex was chosen randomly so as not to bias orientations in further processing. Two types of reconstructions were calculated. For the first reconstruction ('P4 hexamer'), partial signal subtraction was used to remove the contribution of unwanted NC density in the particle images ${ }^{53}$. We modified our earlier localized reconstruction method ${ }^{17}$ to allow subtracting 'all-but-one' P4 hexamer density (available from http://github.com/OPIC-Oxford/localrec), in addition to the other unwanted densities (P1 and P8 shells and RNA) from the icosahedral reconstruction. Only subparticles from the edge of the particle (total of 103,482; maximum $40^{\circ}$ deviation from a side view) were chosen for further analysis to reduce the overlap of the projected hexamer density with the RNA component of the virion that could not be accurately subtracted from the particle images. The structure of the P4 hexamer was then determined using Relion and 3D classification, using a localized reconstruction of the hexamer, calculated from a subset of subparticles and filtered to $40 \AA$ resolution, as a starting model. $3 \mathrm{D}$ classification revealed the hexamer in different orientations relative to the five-fold vertex. Particles from classes showing clear hexamer density were combined and six-fold symmetry was applied on the reconstruction. For the second reconstruction ('NC vertex'), subparticles extracted from the original particle images and orientation parameters from the $\mathrm{P} 4$ hexamer reconstruction were used and no symmetry was applied.

Resolutions of the icosahedral NC reconstruction and P4 hexamer localized reconstruction were estimated by using FSC using 0.143 cutoff. The NC vertex reconstruction was low-pass filtered to the resolution of $\mathrm{P} 4$ hexamer reconstruction $(9.1 \AA)$ and both localized reconstructions were sharpened by applying an inverse $\mathrm{B}$-factor of $-600 \AA^{2}$ (determined by trial and error). Local resolution of the $\mathrm{NC}$ reconstruction was estimated using ResMap ${ }^{54}$. Reconstruction statistics are summarized in Table 2.

Atomic model building and refinement. As no P8 atomic model was available, a polyalanine model was built de novo using Coot. Visually identified densities 


\begin{tabular}{|c|c|c|}
\hline Parameter & P1 & P8 \\
\hline \multicolumn{3}{|l|}{ Refinement } \\
\hline Resolution $(\AA)$ & \multicolumn{2}{|c|}{4.0} \\
\hline Map CC & \multicolumn{2}{|c|}{0.715} \\
\hline \multicolumn{3}{|l|}{ RMS deviations } \\
\hline Bonds $(\AA)$ & \multicolumn{2}{|c|}{0.09} \\
\hline Angles $\left({ }^{\circ}\right)$ & \multicolumn{2}{|c|}{1.0} \\
\hline \multicolumn{3}{|l|}{ Molprobity validation } \\
\hline Clashscore, all atoms (percentile) & 13.8 (55th) & 10.4 (70th) \\
\hline Molprobity score & 1.92 (80th) & 1.53 (94th) \\
\hline Favoured rotamers (poor) (\%) & $98.4(0.1)$ & $97.3(0.0)$ \\
\hline Ramachandran (\% favoured) & 96 & 99.0 \\
\hline Ramachandran (\% allowed) & 99.7 & 100 \\
\hline Ramachandran Plot (\% outliers) & 0.3 & 0 \\
\hline
\end{tabular}

RMS, root mean square.

for bulky residues facilitated building the full atomic model of P8 (L3-Y147) and the C terminus of P4 (R292-L332) with side-chains. The crystal structure of $\phi 6 \mathrm{P} 1$ (PDB:4K7H) was fitted in the $\mathrm{NC}$ map using $\mathrm{COOT}^{55}$ as a rigid body in two different positions corresponding to subunits $\mathrm{P} 1_{\mathrm{A}}$ and $\mathrm{P} 1_{\mathrm{B}} . \mathrm{P} 1_{\mathrm{A}}$ and $\mathrm{P} 1_{\mathrm{B}}$ main-chains and side-chains were adjusted using manual and real space fitting in COOT. The crystal structure of $\$ 6 \mathrm{P} 4$ (PDB:4BLO) ${ }^{19}$ was fitted in the $\mathrm{P} 4$ hexamer and NC vertex localized reconstructions as a rigid body in UCSF Chimera $^{56}$.

Atomic models for all of the chains in the asymmetric unit $\left(\mathrm{P} 1_{\mathrm{A}}, \mathrm{P} 1_{\mathrm{B}}, \mathrm{P} 4\right.$ $\mathrm{C}$ terminus and ten copies of $\mathrm{P} 8$ ) were refined in Phenix.real_space_refine applying secondary structure, rotamer, and Ramachandran plot restraints. In addition, non-crystallographic symmetry restraints were applied on P8 chains ${ }^{57}$. The models were validated with MOLPROBITY ${ }^{58}$. Atomic model refinement statistics are summarized in Table 3. Disordered regions in P4 sequence (UniProt:P11125) were predicted with RONN ${ }^{35}$ and changes in subunit-subunit angles calculated in UCSF Chimera ${ }^{59}$.

Data availability. Density maps and atomic models that support the findings of this study have been deposited in the Electron Microscopy Database and in the Protein Databank with the accession codes EMD-3571 (NC reconstruction), PDB 5MUU (NC atomic model), EMD-3572 (P4 hexamer reconstruction), PDB 5MUV (P4 atomic model fitted in the P4 hexamer reconstruction), EMD-3573 (NC vertex reconstruction), and PDB 5MUW (P4 atomic model fitted in the NC vertex reconstruction).

\section{References}

1. Aksyuk, A. A. \& Rossmann, M. G. Bacteriophage assembly. Viruses 3, 172-203 (2011).

2. Zlotnick, A., Porterfield, J. Z. \& Wang, J. C.-Y. To build a virus on a nucleic acid substrate. Biophys. J. 104, 1595-1604 (2013).

3. Perlmutter, J. D. \& Hagan, M. F. Mechanisms of virus assembly. Annu. Rev. Phys. Chem. 66, 217-239 (2015).

4. Bamford, D. H. Do viruses form lineages across different domains of life? Res. Microbiol. 154, 231-236 (2003).

5. Abrescia, N. G. A., Bamford, D. H., Grimes, J. M. \& Stuart, D. I. Structure unifies the viral universe. Annu. Rev. Biochem. 81, 795-822 (2012).

6. Shepherd, C. M. \& Reddy, V. S. Extent of protein-protein interactions and quasi-equivalence in viral capsids. Proteins 58, 472-477 (2005).

7. Wikoff, W. R. et al. Topologically linked protein rings in the bacteriophage HK97 capsid. Science 289, 2129-2133 (2000).

8. Bennett, M. J., Schlunegger, M. P. \& Eisenberg, D. 3D domain swapping: a mechanism for oligomer assembly. Protein Sci. 4, 2455-2468 (1995).

9. Liu, Y. \& Eisenberg, D. 3D domain swapping: as domains continue to swap. Protein Sci. 11, 1285-1299 (2002).

10. Simpson, A. A., Chipman, P. R., Baker, T. S., Tijssen, P. \& Rossmann, M. G. The structure of an insect parvovirus (Galleria mellonella densovirus) at $3.7 \AA$ Å resolution. Structure 6, 1355-1367 (1998).

11. Tate, J. et al. The crystal structure of cricket paralysis virus: the first view of a new virus family. Nat. Struct. Biol 6, 765-774 (1999).

12. Qu, C. et al. 3D domain swapping modulates the stability of members of an icosahedral virus group. Structure 8, 1095-1103 (2000).

13. Silva, A. M. \& Rossmann, M. G. Refined structure of southern bean mosaic virus at $2.9 \AA$ resolution. J. Mol. Biol. 197, 69-87 (1987).
14. Semancik, J. S., Vidaver, A. K. \& Van Etten, J. L. Characterization of segmented double-helical RNA from bacteriophage ф6. J. Mol. Biol. 78, 617-625 (1973).

15. Butcher, S. J., Dokland, T., Ojala, P. M., Bamford, D. H. \& Fuller, S. D. Intermediates in the assembly pathway of the double-stranded RNA virus $\phi 6$. EMBO J. 16, 4477-4487 (1997).

16. Butcher, S. J., Grimes, J. M., Makeyev, E. V., Bamford, D. H. \& Stuart, D. I. A mechanism for initiating RNA-dependent RNA polymerization. Nature 410, 235-240 (2001).

17. Ilca, S. L. et al. Localized reconstruction of subunits from electron cryomicroscopy images of macromolecular complexes. Nat. Commun. 6, 8843 (2015).

18. Pirttimaa, M. J., Paatero, A. O., Frilander, M. J. \& Bamford, D. H. Nonspecific nucleoside triphosphatase P4 of double-stranded RNA bacteriophage $\phi 6$ is required for single-stranded RNA packaging and transcription. J. Virol. 76, 10122-10127 (2002).

19. El Omari, K. et al. Tracking in atomic detail the functional specializations in viral RecA helicases that occur during evolution. Nucleic Acids Res. 41, 9396-9410 (2013).

20. Poranen, M. M., Butcher, S. J., Simonov, V. M., Laurinmäki, P. \& Bamford, D. H. Roles of the minor capsid protein $\mathrm{P} 7$ in the assembly and replication of double-stranded RNA bacteriophage $\phi 6$. J. Mol. Biol. 383, 529-538 (2008).

21. Nemecek, D., Qiao, J., Mindich, L., Steven, A. C. \& Heymann, J. B. Packaging accessory protein P7 and polymerase P2 have mutually occluding binding sites inside the bacteriophage $\phi 6$ procapsid. J. Virol. 86, 11616-11624 (2012).

22. Nemecek, D. et al. Stepwise expansion of the bacteriophage $\phi 6$ procapsid: possible packaging intermediates. J. Mol. Biol. 414, 260-271 (2011).

23. Huiskonen, J. T. et al. Structure of the bacteriophage $\phi 6$ nucleocapsid suggests a mechanism for sequential RNA packaging. Structure 14, 1039-1048 (2006).

24. Stitt, B. L. \& Mindich, L. Morphogenesis of bacteriophage $\phi 6$ : a presumptive viral membrane precursor. Virology 127, 446-458 (1983).

25. Jäälinoja, H. T., Huiskonen, J. T. \& Butcher, S. J. Electron cryomicroscopy comparison of the architectures of the enveloped bacteriophages $\phi 6$ and $\phi 8$. Structure 15, 157-167 (2007).

26. Poranen, M. M., Paatero, A. O., Tuma, R. \& Bamford, D. H. Self-assembly of a viral molecular machine from purified protein and RNA constituents. Mol. Cell 7, 845-854 (2001).

27. Zhang, X., Jin, L., Fang, Q., Hui, W. H. \& Zhou, Z. H. 3.3 A cryo-EM structure of a nonenveloped virus reveals a priming mechanism for cell entry. Cell 141, 472-482 (2010).

28. Dryden, K. A. et al. Early steps in reovirus infection are associated with dramatic changes in supramolecular structure and protein conformation: analysis of virions and subviral particles by cryoelectron microscopy and image reconstruction. J. Cell Biol. 122, 1023-1041 (1993).

29. Grimes, J. M. et al. The atomic structure of the bluetongue virus core. Nature 395, 470-478 (1998).

30. Zhang, X. et al. Atomic model of a nonenveloped virus reveals $\mathrm{pH}$ sensors for a coordinated process of cell entry. Nat. Struct. Mol. Biol. 23, 74-80 (2016).

31. Zhou, Z. H. et al. Electron cryomicroscopy and bioinformatics suggest protein fold models for rice dwarf virus. Nat. Struct. Biol. 8, 868-873 (2001).

32. Nakagawa, A. et al. The atomic structure of rice dwarf virus reveals the self-assembly mechanism of component proteins. Structure 11, 1227-1238 (2003).

33. Settembre, E. C., Chen, J. Z., Dormitzer, P. R., Grigorieff, N. \& Harrison, S. C. Atomic model of an infectious rotavirus particle. EMBO J. 30, 408-416 (2011).

34. Mohd Jaafar, F. et al. The structure and function of the outer coat protein VP9 of Banna virus. Structure 13, 17-28 (2005).

35. Yang, Z. R., Thomson, R., McNeil, P. \& Esnouf, R. M. RONN: the bio-basis function neural network technique applied to the detection of natively disordered regions in proteins. Bioinformatics 21, 3369-3376 (2005).

36. de Haas, F., Paatero, A. O., Mindich, L., Bamford, D. H. \& Fuller, S. D. A symmetry mismatch at the site of RNA packaging in the polymerase complex of dsRNA bacteriophage $\phi 6$. J. Mol. Biol. 294, 357-372 (1999).

37. Huiskonen, J. T., Jäälinoja, H. T., Briggs, J. A. G., Fuller, S. D. \& Butcher, S. J. Structure of a hexameric RNA packaging motor in a viral polymerase complex. J. Struct. Biol. 158, 156-164 (2007).

38. Liu, H., Wu, L. \& Zhou, Z. H. Model of the trimeric fiber and its interactions with the pentameric penton base of human adenovirus by cryo-electron microscopy. J. Mol. Biol. 406, 764-774 (2011).

39. Nemecek, D. et al. Subunit folds and maturation pathway of a dsRNA virus capsid. Structure 21, 1374-1383 (2013). 
40. Luque, D. et al. The $\mathrm{T}=1$ capsid protein of Penicillium chrysogenum virus is formed by a repeated helix-rich core indicative of gene duplication. J. Virol. 84, 7256-7266 (2010).

41. Paatero, A. O., Mindich, L. \& Bamford, D. H. Mutational analysis of the role of nucleoside triphosphatase $\mathrm{P} 4$ in the assembly of the RNA polymerase complex of bacteriophage $\phi 6$. J. Virol. 72, 10058-10065 (1998).

42. Ojala, P. M., Romantschuk, M. \& Bamford, D. H. Purified $\phi 6$ nucleocapsids are capable of productive infection of host cells with partially disrupted outer membranes. Virology 178, 364-372 (1990).

43. Cvirkaite-Krupovic, V., Poranen, M. M. \& Bamford, D. H. Phospholipids act as secondary receptor during the entry of the enveloped, double-stranded RNA bacteriophage $\phi 6$. J. Gen. Virol. 91, 2116-2120 (2010).

44. Tuma, R., Bamford, J. K., Bamford, D. H. \& Thomas, G. J. Assembly dynamics of the nucleocapsid shell subunit (P8) of bacteriophage $\phi 6$. Biochemistry 38, 15025-15033 (1999).

45. Rousseau, F., Schymkowitz, J. W., Wilkinson, H. R. \& Itzhaki, L. S. Threedimensional domain swapping in p13sucl occurs in the unfolded state and is controlled by conserved proline residues. Proc. Natl Acad. Sci. USA 98, 5596-5601 (2001).

46. Gil-Carton, D. et al. Insight into the assembly of viruses with vertical single $\beta$-barrel major capsid proteins. Structure 23, 1866-1877 (2015).

47. Olkkonen, V. M., Ojala, P. M. \& Bamford, D. H. Generation of infectious nucleocapsids by in vitro assembly of the shell protein on to the polymerase complex of the dsRNA bacteriophage $\phi 6$. J. Mol. Biol. 218, 569-581 (1991).

48. Vidaver, A. K., Koski, R. K. \& Van Etten, J. L. Bacteriophage ф6: a lipidcontaining virus of Pseudomonas phaseolicola. J. Virol. 11, 799-805 (1973)

49. Bamford, D. H., Ojala, P. M., Frilander, M., Walin, L. \& Bamford, J. K. H. in Methods in Molecular Genetics Vol. 6 (ed. Adolph, K. W.) 455-474 (Academic Press, 1995).

50. Li, X. et al. Electron counting and beam-induced motion correction enable near-atomic-resolution single-particle cryo-EM. Nat. Methods 10, 584-590 (2013).

51. Mindell, J. A. \& Grigorieff, N. Accurate determination of local defocus and specimen tilt in electron microscopy. J. Struct. Biol. 142, 334-347 (2003).

52. Scheres, S. H. W. RELION: implementation of a Bayesian approach to cryo-EM structure determination. J. Struct. Biol. 180, 519-530 (2012).

53. Bai, X.-C., Rajendra, E., Yang, G., Shi, Y. \& Scheres, S. H. Sampling the conformational space of the catalytic subunit of human $\gamma$-secretase. Elife 4, e11182 (2015).

54. Kucukelbir, A., Sigworth, F. J. \& Tagare, H. D. Quantifying the local resolution of cryo-EM density maps. Nat. Methods 11, 63-65 (2014).

55. Emsley, P. \& Cowtan, K. Coot: model-building tools for molecular graphics. Acta Crystallogr. D Biol. Crystallogr. 60, 2126-2132 (2004).

56. Goddard, T. D., Huang, C. C. \& Ferrin, T. E. Visualizing density maps with UCSF Chimera. J. Struct. Biol. 157, 281-287 (2007).

57. Adams, P. D. et al. PHENIX: a comprehensive Python-based system for macromolecular structure solution. Acta Crystallogr. D Biol. Crystallogr. 66, 213-221 (2010)

58. Davis, I. W. et al. MolProbity: all-atom contacts and structure validation for proteins and nucleic acids. Nucleic Acids Res. 35, W375-W383 (2007).
59. Goddard, T. D., Huang, C. C. \& Ferrin, T. E. Software extensions to UCSF chimera for interactive visualization of large molecular assemblies. Structure 13, 473-482 (2005).

\section{Acknowledgements}

We thank Alistair Siebert for electron microscopy support and Riitta Tarkiainen for technical help. The OPIC electron microscopy facility was founded by a Wellcome Trust JIF award (060208/Z/00/Z) and is supported by a WT equipment grant $(093305 / \mathrm{Z} / 10 / \mathrm{Z})$ The EU ESFRI Instruct Centre for Virus Production (ICVIR) facility used in this study is supported by the Academy of Finland (grant 272853) and University of Helsinki and this is a collaboration with the Oxford Instruct Centre. This work was funded by Academy of Finland (218080 and 263677 to J.T.H; 283192, 250113, and 272507 to M.M.P.), Sigrid Jusélius Foundation, Medical Research Council (MR/N00065X/1 to K.E.O.), European Research Council under the European Union's Horizon 2020 research and innovation programme (649053 to J.T.H.) and Wellcome Trust Core Award Grant Number 090532/ $\mathrm{Z} / 09 / \mathrm{Z}$. The authors acknowledge the support of employees and the use of experimental resources of Instruct, a Landmark ESFRI project.

\section{Author contributions}

Z.S., S.L.I. and J.T.H. collected and analysed cryo-EM data. K.E.O., M.M.P., D.I.S. and J.T.H. designed the study. X.S. prepared the samples. Z.S., K.E.O. and A.K. built and refined the atomic models. Z.S. and J.T.H. wrote the manuscript and all authors read and commented on the manuscript.

\section{Additional information}

Supplementary Information accompanies this paper at http://www.nature.com/ naturecommunications

Competing interests: The authors declare no competing financial interests.

Reprints and permission information is available online at http://npg.nature.com/ reprintsandpermissions/

How to cite this article: Sun, Z. et al. Double-stranded RNA virus outer shell assembly by bona fide domain-swapping. Nat. Commun. 8, 14814 doi: 10.1038/ncomms14814 (2017).

Publisher's note: Springer Nature remains neutral with regard to jurisdictional claims in published maps and institutional affiliations

(c) (i) This work is licensed under a Creative Commons Attribution 4.0 International License. The images or other third party material in this article are included in the article's Creative Commons license, unless indicated otherwise in the credit line; if the material is not included under the Creative Commons license, users will need to obtain permission from the license holder to reproduce the material. To view a copy of this license, visit http://creativecommons.org/licenses/by/4.0/

C The Author(s) 2017 